This postprint is published in:

Analysis and applications, Volume 13, Issue 6, 2015, 645-661

DOI: $10.1142 / S 0219530514500420$

\title{
STATIONARY WAVES OF SCHRÖDINGER-TYPE EQUATIONS WITH VARIABLE EXPONENT
}

\author{
DUŠAN REPOVŠ
}

\begin{abstract}
We are concerned with a class of nonlinear Schrödinger-type equations with a reaction term and a differential operator that involves a variable exponent. By using related variational methods, we establish several existence results.
\end{abstract}

\section{INTRODUCTION}

The topic of function spaces with variable exponents has undergone an impressive development over the last decades. It seems that the oldest references in this field are the works by Orlicz [20] and Nakano [19]. This impressive revival is essentially connected with relevant applications to nonlinear partial differential equations and fluid dynamics.

The field we are concerned with in this work is of central interest, since the Schrödinger equation plays in quantum mechanics the same role as the Newton laws of conservation of energy play in classical mechanics. Schrödinger gave the classical derivation of this basic equation, based upon the analogy between mechanics and optics, and using the developments due to Louis de Broglie. The linear Schrödinger equation provides a thorough description of a particle in a non-relativistic setting. The structure of the nonlinear Schrödinger equation is much more complicated. The nonlinear Schrödinger equation describes central phenomena arising in nonlinear optics, Bose-Einstein condensates, Heisenberg ferromagnets and magnons, plasma physics (the Kurihara superfluid film equation or Langmuir waves), condensed matter theory, etc. We refer to Ablowitz, Prinari and Trubatch [1] and Sulem [25] for a modern overview, including relevant applications.

In this paper, we study a Schrödinger-type equation in the framework of function spaces with variable exponent. Problems of this type have been intensively studied in the last few years due to major applications to non-Newtonian electrorheological fluids (Halsey [13], Ruzicka [22]) or image restoration (Chen, Levine and Rao [5]). Our main purpose is to extend the study of Laplace-type operators to more general classes of equations with variable exponent and nonhomogeneous differential operator. We are concerned with Schrödingertype equations that involve the differential operator $\operatorname{div}(A(x,|\nabla u|) \nabla u)$ and power-type nonlinearities with exponent variable. The abstract setting in the present paper corresponds to very general differential operators that include the usual $p(x)$-Laplace or $p(x)$-mean

Key words and phrases. Lebesgue-Sobolev spaces with variable exponent; Fountain theorem; Mountain Pass geometry; Leray-Lions operators; hemivariational inequality; resonance.

2010 Mathematics Subject Classification. 35J62, 35J70, 46E35, 58E05, 35M86, 47J20. 
curvature operators, resp.

$$
\operatorname{div}\left(|\nabla u|^{p(x)-2} \nabla u\right), \quad \operatorname{div}\left(p(x)|\nabla u|^{p(x)-2} \nabla u\right) \quad \text { and } \quad \operatorname{div}\left(\left(1+|\nabla u|^{2}\right)^{(p(x)-2) / 2} \nabla u\right) .
$$

An excellent reference for the most significant mathematical methods employed in this paper is the book by Ciarlet [6].

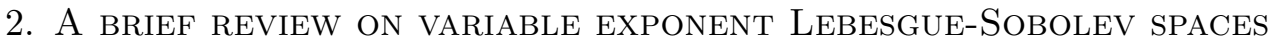

Throughout this paper we assume that $\Omega$ is a bounded open set in $\mathbb{R}^{N}$ with smooth boundary.

In this section, we recall some definitions and basic properties of the variable exponent Lebesgue space $L^{p(x)}(\Omega)$ and $W_{0}^{1, p(x)}(\Omega)$. Roughly speaking, Lebesgue and Sobolev spaces with variable exponent are functional spaces of Lebesgue's and Sobolev's type in which different space directions have different roles. The variable exponent Lebesgue space $L^{p(x)}(\Omega)$ is a special case of Orlicz-Musielak spaces treated by Musielak [18].

Denote

$$
C_{+}(\bar{\Omega})=\{h ; h \in C(\bar{\Omega}), h(x)>1, \text { for all } x \in \bar{\Omega}\} .
$$

For any $h \in C_{+}(\bar{\Omega})$, we define

$$
h^{+}=\max \{h(x) ; x \in \bar{\Omega}\}, \quad h^{-}=\min \{h(x) ; x \in \bar{\Omega}\} .
$$

For any $p \in C_{+}(\bar{\Omega})$, we define the variable exponent Lebesgue space

$$
L^{p(x)}(\Omega)=\left\{u: \Omega \rightarrow \mathbb{R} ; u \text { is measurable and } \int_{\Omega}|u(x)|^{p(x)} d x<\infty\right\},
$$

endowed with the Luxemburg norm

$$
|u|_{L^{p(x)}(\Omega)}=|u|_{p(x)}=\inf \left\{\mu>0 ; \int_{\Omega}\left|\frac{u(x)}{\mu}\right|^{p(x)} d x \leq 1\right\} .
$$

Then $\left(L^{p(x)}(\Omega),|\cdot|_{p(x)}\right)$ is a Banach space, cf. [14].

As established in [9], $\left(L^{p(x)}(\Omega),|\cdot|_{p(x)}\right)$ is a separable, uniformly convex Banach space and its dual space is $L^{q(x)}(\Omega)$, where $\frac{1}{p(x)}+\frac{1}{q(x)}=1$. Moreover, for all $u \in L^{p(x)}(\Omega)$ and $v \in L^{q(x)}(\Omega)$, we have the Hölder inequality

$$
\left|\int_{\Omega} u v d x\right| \leq\left(\frac{1}{p^{-}}+\frac{1}{q^{-}}\right)|u|_{p(x)}|v|_{q(x)} .
$$

If $p_{1}(x), p_{2}(x) \in C_{+}(\bar{\Omega})$ and $p_{1}(x) \leq p_{2}(x)$ for all $x \in \bar{\Omega}$, then $L^{p_{2}(x)}(\Omega) \hookrightarrow L^{p_{1}(x)}(\Omega)$ and the embedding is continuous.

An important role in manipulating the generalized Lebesgue space is played by the $p(x)$ modular of the $L^{p(x)}(\Omega)$ space, which is the mapping $\rho_{p(x)}: L^{p(x)}(\Omega) \rightarrow \mathbb{R}$ defined by

$$
\rho_{p(x)}(u)=\int_{\Omega}|u|^{p(x)} d x .
$$

Proposition 2.1 (See [11]). For $u \in L^{p(x)}(\Omega)$ and $u_{n} \subset L^{p(x)}(\Omega)$, we have

(1) $|u|_{p(x)}<1 \quad$ (respectively $\left.=1 ;>1\right) \Longleftrightarrow \rho_{p(x)}(u)<1 \quad$ (respectively $\left.=1 ;>1\right)$;

(2) for $u \neq 0,|u|_{p(x)}=\lambda \Longleftrightarrow \rho_{p(x)}\left(\frac{u}{\lambda}\right)=1$; 
(3) if $|u|_{p(x)}>1$, then $|u|_{p(x)}^{p^{-}} \leq \rho_{p(x)}(u) \leq|u|_{p(x)}^{p^{+}}$;

(4) if $|u|_{p(x)}<1$, then $|u|_{p(x)}^{p^{+}} \leq \rho_{p(x)}(u) \leq|u|_{p(x)}^{p^{-}}$;

(5) $\left|u_{n}-u\right|_{p(x)} \rightarrow 0$ (respectively $\left.\rightarrow \infty\right) \Longleftrightarrow \rho_{p(x)}\left(u_{n}-u\right) \rightarrow 0$ (respectively $\left.\rightarrow \infty\right)$, since $p^{+}<\infty$.

The Sobolev space with variable exponent $W^{1, p(x)}(\Omega)$ is defined by

$$
W^{1, p(x)}(\Omega)=\left\{u \in L^{p(x)}(\Omega) ; \partial_{x_{i}} u \in L^{p(x)}(\Omega), i \in\{1, \ldots, N\}\right\} .
$$

If equipped with the norm

$$
\|u\|_{W^{1, p(x)}(\Omega)}=|u|_{L^{p(x)}(\Omega)}+\sum_{i=1}^{N}\left|\partial_{x_{i}} u\right|_{L^{p(x)}(\Omega)},
$$

then $\left(W^{1, p(x)}(\Omega),\|\cdot\|_{W^{1, p(x)}(\Omega)}\right)$ is a separable and reflexive Banach space (see $[14$, Theorem $1.3])$.

As observed by Zhikov [28] in relationship with the Lavrentiev phenomenon, it is possible that minimizers of certain variational integrals are not smooth. The following log-Hölder condition was first used in the variable exponent context by Zhikov [27]. More precisely, we say that a function $h: \Omega \rightarrow \mathbb{R}$ is log-Hölder continuous on $\Omega$ if there exists $C>0$ such that

$$
|h(x)-h(y)| \leq-\frac{C}{\log |x-y|} \quad \text { for all } x, y \in \Omega,|x-y| \leq \frac{1}{2}
$$

As established in [9] (see also [7, Theorem 9.1.8]), if $\Omega$ is bounded with Lipschitz boundary and $p$ is log-Hölder continuous, then $C^{\infty}(\bar{\Omega})$ is dense in $W^{1, p(x)}(\Omega)$. We point out that though log-Hölder continuity of $p(x)$ is sufficient to imply the density of test functions in $W^{1, p(x)}$, this condition is far from being necessary. For instance, Edmunds and Rákosník [8] derived the same conclusion under a local monotony condition on $p$.

Let $p$ be log-Hölder continuous. The Sobolev space $W_{0}^{1, p(x)}(\Omega)$ with zero boundary values is the closure of the set of $W^{1, p(x)}(\Omega)$-functions with compact support. Furthermore, if $p$ is bounded then $W_{0}^{1, p(x)}(\Omega)$ is the closure of $C_{0}^{\infty}(\Omega)$ in the space $W^{1, p(x)}(\Omega)$, see [7, Proposition 11.2.3]. The norm $\|u\|=\sum_{i=1}^{N}\left|\partial_{x_{i}} u\right|_{p(x)}$ is an equivalent norm in $W_{0}^{1, p(x)}(\Omega)$ (see [16]). Hence $W_{0}^{1, p(x)}(\Omega)$ is a separable and reflexive Banach space. Note that when $s \in C_{+}(\bar{\Omega})$ and $s(x)<p^{*}(x)$ for all $x \in \bar{\Omega}$, where $p^{*}(x)=\frac{N p(x)}{N-p(x)}$ if $p(x)<N$ and $p^{*}(x)=\infty$ if $p(x) \geq N$, then the embedding $W_{0}^{1, p(x)}(\Omega) \hookrightarrow L^{s(x)}(\Omega)$ is compact.

\section{MAIN RESUlts}

In this paper we study the following nonlinear problem

$$
\begin{cases}-\operatorname{div}(A(x,|\nabla u|) \nabla u)=f(x, u) & \text { if } x \in \Omega \\ u=0 & \text { if } x \in \partial \Omega .\end{cases}
$$

Problems of this type are motivated by models in mathematical physics (see Reed and Simon [21] and Strauss [24]), where certain stationary waves in nonlinear Schrödinger or 
Klein-Gordon equations can be reduced to this form. Equation (2) arises in the study of the Schrödinger-type equation

$$
i v_{t}-\operatorname{div}(A(x,|\nabla v|) \nabla v)=f(x, v)
$$

when looking for standing waves, that is, solutions of the type $v(x, t)=e^{-i c t} u(x)$, where $c$ is a real constant. This problem has a central role in quantum mechanics for the study of particles of stochastic fields modelled by Lévy processes. A path integral over the Lévy flights paths and a nonlinear Schrödinger equation is formulated by Laskin [15] from the idea of Feynman and Hibbs's paths integrals.

Fix $p \in C_{+}(\bar{\Omega})$ and suppose that $p$ is log-Hölder continuous.

We assume that $A: \Omega \times[0, \infty) \rightarrow[0, \infty)$ satisfies the following hypotheses:

(A1) the mapping $\Omega \ni x \longmapsto A(x, s)$ is measurable for all $s \geq 0$ and the mapping $(0, \infty) \ni$ $s \longmapsto A(x, s)$ is absolutely continuous for a.a. $x \in \Omega$;

(A2) there exist $a_{1} \in L^{p^{\prime}(x)}(\Omega)$ and $a_{2}>0$ such that

$$
|A(x,|z|) z| \leq a_{1}(x)+a_{2}|z|^{p(x)-1} \quad \text { a.e. } x \in \Omega \text {, all } z \in \mathbb{R}^{N}
$$

(A3) there exists $a_{3}>0$ such that for a.a. $x \in \Omega$ and for all $s>0$

$$
\min \left\{A(x, s), A(x, s)+s \partial_{s} A(x, s)\right\} \geq a_{3} \min \left\{1, s^{p(x)-2}\right\} .
$$

(A4) we have $t^{2} A(x, t) \leq p_{+} \int_{0}^{t} s A(x, s) d s$ for a.a. $x \in \Omega$ and for all $t \in(0, \infty)$.

We assume that the nonlinear term $f: \Omega \times \mathbb{R} \rightarrow \mathbb{R}$ is a Carathéodory function satisfying the following conditions:

(f1) there exists $C>0$ such that $|f(x, t)| \leq C|t|^{q(x)-1}$ for a.a. $x \in \Omega$ and for all $t \in \mathbb{R}$, where $q \in C_{+}(\bar{\Omega})$ and $\max _{x \in \bar{\Omega}} p(x)<\min _{x \in \bar{\Omega}} q(x), q(x)<p^{*}(x)$ for all $x \in \bar{\Omega}$;

(f2) there exist $\mu>p_{+}$and $R>0$ such that

$$
0<\mu F(x, t) \leq t f(x, t) \quad \text { for all } x \in \Omega \text { and for all } t \geq R,
$$

where $F(x, t):=\int_{0}^{t} f(x, s) d s$;

(f3) $\lim _{t \rightarrow 0} f(x, t) /|t|^{p_{+}-1}=0$ uniformly for $x \in \Omega$.

We say that $u$ is a solution of problem (2) if $u \in W_{0}^{1, p(x)}(\Omega) \backslash\{0\}$ and

$$
\int_{\Omega} A(x,|\nabla u|) \nabla u \cdot \nabla v d x=\lambda \int_{\Omega} f(x, u) v d x \quad \text { for all } v \in W_{0}^{1, p(x)}(\Omega) .
$$

The first result in this paper establishes the existence of solutions to problem (2) under the above hypotheses. This result extends previous existence properties to a very large class of nonlinear differential operators. The proof combines variational arguments and related energy estimates. A key role is played by the Mountain Pass Theorem, see Ambrosetti and Rabinowitz [2].

Theorem 3.1. Assume that $A$ and $f$ satisfy conditions (A1)-(A4) and (f1)-(f3). Then problem (2) has at least one solution. 
Next, we are concerned with the existence of multiple high-energy solutions of problem (2). For this purpose, we consider the associated energy functional $\mathcal{E}: W_{0}^{1, p(x)}(\Omega) \rightarrow \mathbb{R}$ defined by

$$
\mathcal{E}(u)=\int_{\Omega}\left(\int_{0}^{|\nabla u(x)|} s A(x, s) d s\right) d x-\int_{\Omega} F(x, u) d x .
$$

Assuming that $f(x, \cdot)$ is odd, we prove that problem (2) has a sequence of solutions with higher and higher energies. The statement of this result is the following.

Theorem 3.2. Suppose that hypotheses (A1)-(A4) and (f1)-(f2) are fulfilled and $f(x,-t)=$ $-f(x, t)$ for a.a. $x \in \Omega$ and all $t \in \mathbb{R}$. Then problem (2) admits a sequence of solutions $\left(u_{n}\right)$ such that $\mathcal{E}\left(u_{n}\right) \rightarrow+\infty$ as $n \rightarrow \infty$.

A central role in the proof of Theorem 3.2 is played by the Fountain Theorem, which is due to Bartsch [4]. This result is nicely presented in Willem [26] by using the quantitative deformation lemma. We also point out that the dual version of the Fountain Theorem is due to Bartsch and Willem, see [26]. It should be noted that the Palais-Smale condition plays an important role for these theorems and their applications.

A related question concerns the minimization problem corresponding to the associated Rayleigh quotient, namely

$$
\lambda_{1}:=\inf _{u \in W_{0}^{1, p(x)}(\Omega) \backslash\{0\}} \frac{\int_{\Omega}\left(\int_{0}^{\mid \nabla u(x)} s A(x, s) d s\right) d x}{\int_{\Omega} F(x, u) d x} .
$$

A natural assumption, in order to avoid degeneracies, is the following.

(f4) for all $(x, u) \in \Omega \times \mathbb{R}$ we have $u f(x, u) \geq 0$ and $f \not \equiv 0$.

In the case corresponding to the Laplace or $p$-Laplace operators we have $\lambda_{1}>0$. However, it may happen that $\lambda_{1}=0$, see [17]. The next result establishes a sufficient condition such that $\lambda_{1}$ is positive. This condition takes into account the growth of the variable potentials $p(\cdot)$ and $q()$ and is the following.

(A5) we have $2\left(q_{+}-q_{-}\right)<p_{-}$.

Theorem 3.3. Assume that hypotheses (A1)-(A5), (f1) and (f4) are fulfilled. Then

$$
\lambda_{1}:=\inf _{u \in W_{0}^{1, p(x)}(\Omega) \backslash\{0\}} \frac{\int_{\Omega}\left(\int_{0}^{\mid \nabla u(x)} s A(x, s) d s\right) d x}{\int_{\Omega} F(x, u) d x}>0 .
$$

Moreover, for all $\lambda \geq \lambda_{1}$ the nonlinear problem

$$
\begin{cases}-\operatorname{div}(A(x,|\nabla u|) \nabla u)=\lambda f(x, u) & \text { if } x \in \Omega \\ u=0 & \text { if } x \in \partial \Omega\end{cases}
$$

has a nontrivial solution.

In particular, Theorem 3.3 establishes a concentration property at infinity for the spectrum problem $S u=\lambda T u$, where $S u:=-\operatorname{div}(A(x,|\nabla u|) \nabla u)$ and $T u:=f(x, u)$. 


\section{Auxiliary properties}

From now on we assume that $A$ and $f$ satisfy the hypotheses in the previous section.

We first establish that the energy $\mathcal{E}$ is of class $C^{1}$ on $W_{0}^{1, p(x)}(\Omega)$ and for all $u, v \in$ $W_{0}^{1, p(x)}(\Omega)$

$$
\mathcal{E}^{\prime}(u)(v)=\int_{\Omega} A(x,|\nabla u|) \nabla u \nabla v d x-\int_{\Omega} f(x, u) v d x .
$$

Relation (5) follows if we prove that

$$
\mathcal{E}_{0}^{\prime}(u)(v)=\int_{\Omega} A(x,|\nabla u|) \nabla u \cdot \nabla v d x
$$

where

$$
\mathcal{E}_{0}(u)=\int_{\Omega}\left(\int_{0}^{|\nabla u(x)|} s A(x, s) d s\right) d x .
$$

Fix $u, v \in W_{0}^{1, p(x)}(\Omega)$ and $\lambda \in \mathbb{R}$ close to zero, say $0<|\lambda|<1$. Thus, by hypothesis (A2) combined with the mean value theorem, there exists $\theta$ between 0 and $\lambda$ such that

$$
\begin{aligned}
& \frac{\int_{0}^{|\nabla u(x)+\lambda \nabla v(x)|} A(x, s) s d s-\int_{0}^{|\nabla u(x)|} A(x, s) s d s}{\lambda}= \\
& A(x,|\nabla u(x)+\theta \nabla v(x)|)|\nabla u(x)+\theta \nabla v(x)||\nabla v(x)| \leq \\
& \left(a_{1}(x)+a_{2}|\nabla u(x)+\theta \nabla v(x)|^{p(x)-1}\right)|\nabla v(x)| \leq \\
& \leq\left(a_{1}(x)+a_{2}(|\nabla u(x)|+|\nabla v(x)|)^{p(x)-1} \mid\right)|\nabla v(x)| .
\end{aligned}
$$

Next, we show that the right-hand side of relation $(7)$ is in $L^{1}(\Omega)$. Indeed, by Hölder's inequality, we have

$$
\begin{aligned}
& \int_{\Omega}\left(a_{1}(x)+a_{2}(|\nabla u(x)|+|\nabla v(x)|)^{p(x)-1}\right)|\nabla v(x)| d x \leq \\
& 2\left|a_{1}\right|_{p^{\prime}(\cdot)}|\nabla v|_{p(\cdot)}+2 a_{2}\left|(|\nabla u|+|\nabla v|)^{p(x)-1}\right|_{p^{\prime}(\cdot)}|\nabla v|_{p(\cdot)} \leq \\
& 2\left|a_{1}\right|_{p^{\prime}(\cdot)}|\nabla v|_{p(\cdot)}+C\left(\int_{\Omega}\left(|\nabla u(x)|^{p(x)}+|\nabla v(x)|^{p(x)}\right) d x\right)^{1 / p_{-}^{\prime}}|\nabla v|_{p(\cdot)} .
\end{aligned}
$$

Taking $\lambda \rightarrow 0$, we also have $\theta \rightarrow 0$. Thus, by the Lebesgue dominated convergence theorem,

$$
\begin{aligned}
\lim _{\lambda \rightarrow 0} \frac{\mathcal{E}_{0}(u+\lambda v)-\mathcal{E}_{0}(u)}{\lambda} & =\int_{\Omega} \lim _{\theta \rightarrow 0} A(x,|\nabla u+\theta \nabla v|)(\nabla u+\theta \nabla v) \cdot \nabla v d x \\
& =\int_{\Omega} A(x,|\nabla u|) \nabla u \cdot \nabla v d x,
\end{aligned}
$$

which proves our claim (6).

Next, we consider the following operators:

(i) the gradient operator $\nabla: W_{0}^{1, p(x)}(\Omega) \rightarrow L^{p(x)}\left(\Omega, \mathbb{R}^{N}\right)$; 
(ii) the Nemytskii operator $T: L^{p(x)}\left(\Omega, \mathbb{R}^{N}\right) \rightarrow L^{p^{\prime}(x)}\left(\Omega, \mathbb{R}^{N}\right)$ defined by $T u(x)=$ $A(x,|u(x)|) u(x)$ for all $u \in L^{p(x)}\left(\Omega, \mathbb{R}^{N}\right)$;

(iii) the linear operator $L: L^{p^{\prime}(x)}\left(\Omega, \mathbb{R}^{N}\right) \rightarrow W^{-1, p^{\prime}(x)}(\Omega)$ defined by

$$
L u(v)=\int_{\Omega} u(x) \cdot \nabla v(x) d x \quad \text { for all } u \in L^{p^{\prime}(x)}\left(\Omega, \mathbb{R}^{N}\right) \text { and } v \in W_{0}^{1, p(x)}(\Omega) .
$$

These operators are continuous and $\mathcal{E}_{0}^{\prime}=L \circ T \circ \nabla$, hence $\mathcal{E}_{0}$ is of class $C^{1}$.

The next result is a counter-part of formula (2.2) in Simon [23]. More precisely, in [23] it is established that for all $\xi, \zeta \in \mathbb{R}^{N}$

$$
|\xi-\zeta|^{p} \leq \begin{cases}c\left(|\xi|^{p-2} \xi-|\zeta|^{p-2} \zeta\right)(\xi-\zeta) & \text { for } \quad p \geq 2 \\ c\left\langle|\xi|^{p-2} \xi-|\eta|^{p-2} \eta, \xi-\eta\right\rangle^{p / 2}\left(|\xi|^{p}+|\eta|^{p}\right)^{(2-p) / 2} & \text { for } 1<p<2,\end{cases}
$$

where $c$ is a positive constant.

As above, we distinguish between the singular case corresponding to $1<p(x)<2$ and the degenerate case, which corresponds to $p(x)>2$. The version of the above inequalities for variable exponents is the following.

Lemma 4.1. Assume that hypotheses (A1) and (A3) are fulfilled. Then there exists a positive constant $C$ such that for all $\xi, \zeta \in \mathbb{R}^{N}$ with $(\xi, \zeta) \neq(0,0)$, we have:

(i) $(A(x,|\xi|) \xi-A(x,|\zeta|) \zeta) \cdot(\xi-\zeta) \geq C_{1}|\xi-\zeta|^{p(x)}$ for all $x \in \Omega$ with $p(x) \geq 2$;

(ii) $(A(x,|\xi|) \xi-A(x,|\zeta|) \zeta) \cdot(\xi-\zeta) \geq C_{2}|\xi-\zeta|^{2} \min \left\{1,(|\xi|+|\zeta|)^{p(x)-2}\right\}$ for all $x \in \Omega$ with $1<p(x)<2$.

Proof. We have

$$
\begin{aligned}
(A(x,|\xi|) \xi-A(x,|\zeta|) \zeta) \cdot(\xi-\zeta) & =\sum_{i=1}^{N}\left(A(x,|\xi|) \xi_{i}-A(x,|\zeta|) \zeta_{i}\right)\left(\xi_{i}-\zeta_{i}\right) \\
& =\sum_{i=1}^{N}\left(\varphi_{i}(x, \xi)-\varphi(x, \zeta)\right)\left(\xi_{i}-\zeta_{i}\right)
\end{aligned}
$$

where $\varphi_{i}(x, w):=A(x,|w|) w_{i}$ for all $w \in \mathbb{R}^{N}$. But

$$
\varphi_{i}(x, \xi)-\varphi(x, \zeta)=\sum_{j=1}^{N} \int_{0}^{1} \frac{\partial \varphi_{i}(x, z)}{\partial z_{j}}\left(\xi_{j}-\zeta_{j}\right) d t,
$$

where $z=\zeta+t(\xi-\zeta)$. Therefore

$$
(A(x,|\xi|) \xi-A(x,|\zeta|) \zeta) \cdot(\xi-\zeta)=\sum_{i, j=1}^{N} \int_{0}^{1} \frac{\partial \varphi_{i}(x, z)}{\partial z_{j}}\left(\xi_{i}-\zeta_{i}\right)\left(\xi_{j}-\zeta_{j}\right) d t .
$$

Fix $z, w \in \mathbb{R}^{N} \backslash\{0\}$. We observe that

$$
\begin{aligned}
\sum_{i, j=1}^{N} \frac{\partial \varphi_{i}(x, z)}{\partial z_{j}} w_{i} w_{j} & =A(x,|z|)|w|^{2}+\frac{1}{|z|} A_{s}(x,|z|)(z \cdot w)^{2} \\
& =|w|^{2}\left(A(x,|z|)+|z| A_{s}(x,|z|)\left(\frac{z}{|z|} \cdot \frac{w}{|w|}\right)^{2}\right) .
\end{aligned}
$$


(i) Assuming that $p(x) \geq 2$, hypothesis (A3) and relation (9) yield

$$
\sum_{i, j=1}^{N} \frac{\partial \varphi_{i}(x, \xi)}{\partial \xi_{j}} \zeta_{i} \zeta_{j} \geq a_{3}|\xi|^{p(x)-2}|\zeta|^{2}
$$

Returning to relation (8) we deduce that for all $t>0$

$$
(A(x,|\xi|) \xi-A(x,|\zeta|) \zeta) \cdot(\xi-\zeta) \geq a_{3} \int_{0}^{1}|\zeta+t(\xi-\zeta)|^{p(x)-2}|\xi-\zeta|^{2} d t .
$$

Choosing $t=1 / 3$ we deduce that

$$
|\zeta+t(\xi-\zeta)| \geq \max \{|\xi|,|\zeta|\}-\frac{|\xi-\zeta|}{3} \geq \frac{|\xi-\zeta|}{3} .
$$

Thus, by (10), we conclude that there exists $C_{1}>0$ such that

$$
(A(x,|\xi|) \xi-A(x,|\zeta|) \zeta) \cdot(\xi-\zeta) \geq C_{1}|\xi-\zeta|^{p(x)} .
$$

(ii) Assume that $1<p(x)<2$. Using assumption (A3) we have

$$
\sum_{i, j=1}^{N} \frac{\partial \varphi_{i}(x, \xi)}{\partial \xi_{j}} \zeta_{i} \zeta_{j} \geq a_{3} \min \left\{1,|\xi|^{p(x)-2}\right\}|\zeta|^{2} .
$$

It follows that

$$
\begin{aligned}
& (A(x,|\xi|) \xi-A(x,|\zeta|) \zeta) \cdot(\xi-\zeta)= \\
& \sum_{i, j=1}^{N} \int_{0}^{1} \frac{\partial \varphi_{i}}{\partial z_{j}}(x, \zeta+t(\xi-\zeta))\left(\xi_{i}-\zeta_{i}\right)\left(\xi_{j}-\zeta_{j}\right) d t \geq \\
& a_{3} \int_{0}^{1} \min \left\{1,|\zeta+t(\xi-\zeta)|^{p(x)-2}\right\}|\xi-\zeta|^{2} d t \geq \\
& C|\xi-\zeta|^{2} \min \left\{1,(|\xi|+|\zeta|)^{p(x)-2}\right\} .
\end{aligned}
$$

This completes the proof.

Remark 4.1. Taking into account the expression of $\mathcal{E}_{0}^{\prime}$ in (6), Lemma 4.1 implies that the operator $\mathcal{E}_{0}^{\prime}: W_{0}^{1, p(x)}(\Omega) \rightarrow W^{-1, p^{\prime}(x)}(\Omega)$ is strictly monotone. This fact is in accordance with the hypotheses imposed in [7, Section 13.4].

\section{Proof of Theorem 3.1}

By the results in the previous section, in order to find a solution of problem (2), it is enough to show that the energy functional $\mathcal{E}$ has a nontrivial critical point. For this purpose we first check that $\mathcal{E}$ has the Mountain Pass geometry and then we show that $\mathcal{E}$ satisfies the Palais-Smale condition.

By hypotheses (f1) and (f3), for all $\varepsilon>0$ there exists $C_{\varepsilon}>0$ such that for all $(x, u) \in$ $\Omega \times \mathbb{R}$

Thus, by (A3) and (A4)

$$
|F(x, u)| \leq \varepsilon|u|^{p_{+}}+C_{\varepsilon}|u|^{q(x)} .
$$

$$
\begin{aligned}
\mathcal{E}(u) & \geq C|\nabla u|_{p(\cdot)}^{p_{+}}-\int_{\Omega}\left(\varepsilon|u|^{p_{+}}+C_{\varepsilon}|u|^{q(x)}\right) d x \\
& \geq\left. C_{1}\left\|\left.u\right|_{W_{0}^{1, p(x)}(\Omega)} ^{p_{+}}-\varepsilon\right\| u\right|_{W_{0}^{1, p(x)}(\Omega)} ^{p_{+}}-C_{2}(\varepsilon)|u|_{q(\cdot)}^{q_{-}} .
\end{aligned}
$$


By (f1) we know that $p_{+}<q_{-}$. This implies that there exists $\varepsilon>0$ small enough and there are positive numbers $r$ and $\rho$ such that $\mathcal{E}(u) \geq \rho$ for all $u \in W_{0}^{1, p(x)}(\Omega)$ with $\|\left. u\right|_{W_{0}^{1, p(x)}(\Omega)}=$ $r$.

We now check the second geometric assumption of the Mountain Pass Theorem, namely the existence of a "valley". Fix $\varphi \in W_{0}^{1, p(x)}(\Omega) \backslash\{0\}$ and $t>0$. We have

$$
\mathcal{E}(t \varphi)=\int_{\Omega}\left(\int_{0}^{t|\nabla \varphi(x)|} s A(x, s) d s\right) d x-\int_{\Omega} F(x, t \varphi) d x .
$$

Hypothesis (f3) implies that there are $\alpha>p_{+}$and positive constants $A, B$ such that for all $(x, u) \in \Omega \times \mathbb{R}$

$$
F(x, u) \geq A|u|^{\alpha}-B .
$$

For fixed $x \in \Omega$ and $z \in \mathbb{R}^{N}$, consider the differentiable function

$$
h(t):=\int_{0}^{t|z|} s A(x, s) d s \quad t>0 .
$$

It follows that $h^{\prime}(t)=t|z|^{2} A(x, t|z|)$. Thus, by (A4),

$$
h^{\prime}(t)=\frac{1}{t}(t|z|)^{2} A(x, t|z|) \leq \frac{p_{+}}{t} \int_{0}^{t|z|} s A(x, s) d s=\frac{p_{+}}{t} h(t) .
$$

By integration we deduce that for all $t>0$

$$
h(t)=\int_{0}^{t|z|} s A(x, s) d s \leq C t^{p_{+}},
$$

where

$$
C=C(x, z)=\int_{0}^{|z|} s A(x, s) d s
$$

Using estimates (12) and (13), relation (11) yields

$$
\mathcal{E}(t \varphi) \leq C(\varphi) t^{p_{+}}-C_{1} t^{\alpha}+C_{2}
$$

where $C(\varphi), C_{1}, C_{2}$ are positive constants. Since $\alpha>p_{+}$, relation (14) shows that $\mathcal{E}(t \varphi)<0$ for $t$ large enough.

Next, we show that $\mathcal{E}$ satisfies the Palais-Smale condition. For this purpose we need the following auxiliary result, which extends a classical property in functional analysis.

Lemma 5.1. Let $\left(u_{n}\right)$ be a sequence in $W_{0}^{1, p(x)}(\Omega)$ that converges weakly to $u$ and such that

$$
\limsup _{n \rightarrow \infty}\left(\mathcal{E}_{0}^{\prime}\left(u_{n}\right)-\mathcal{E}_{0}^{\prime}(u)\right)\left(u_{n}-u\right) \leq 0 .
$$

Then $\left(u_{n}\right)$ converges strongly in $W_{0}^{1, p(x)}(\Omega)$.

Proof. We already know (see Remark 4.1) that $\mathcal{E}_{0}^{\prime}$ is a monotone operator, hence

$$
\left(\mathcal{E}_{0}^{\prime}\left(u_{n}\right)-\mathcal{E}_{0}^{\prime}(u)\right)\left(u_{n}-u\right) \geq 0 .
$$

Therefore

$$
\lim _{n \rightarrow \infty}\left(\mathcal{E}_{0}^{\prime}\left(u_{n}\right)-\mathcal{E}_{0}^{\prime}(u)\right)\left(u_{n}-u\right)=0 .
$$


But

$$
\left(\mathcal{E}_{0}^{\prime}\left(u_{n}\right)-\mathcal{E}_{0}^{\prime}(u)\right)\left(u_{n}-u\right)=\int_{\Omega}\left(A\left(x,\left|\nabla u_{n}\right|\right) \nabla u_{n}-A(x,|\nabla u|) \nabla u\right) \cdot\left(\nabla u_{n}-\nabla u\right) d x .
$$

We claim that

$$
\int_{\Omega}\left|\nabla u_{n}-\nabla u\right|^{p(x)} d x \rightarrow 0 \quad \text { as } n \rightarrow \infty
$$

which implies that $\left(u_{n}\right)$ converges strongly to $u$ in $W_{0}^{1, p(x)}(\Omega)$.

We have

$$
\int_{\Omega}\left|\nabla u_{n}-\nabla u\right|^{p(x)} d x=\int_{\Omega_{+}}\left|\nabla u_{n}-\nabla u\right|^{p(x)} d x+\int_{\Omega_{-}}\left|\nabla u_{n}-\nabla u\right|^{p(x)} d x,
$$

where

$$
\Omega_{+}:=\{x \in \Omega ; p(x) \geq 2\} \quad \Omega_{-}:=\{x \in \Omega ; 1<p(x)<2\} .
$$

Using Lemma 4.1 we obtain

$$
\begin{aligned}
& \int_{\Omega_{+}}\left(A\left(x,\left|\nabla u_{n}\right|\right) \nabla u_{n}-A(x,|\nabla u|) \nabla u\right) \cdot\left(\nabla u_{n}-\nabla u\right) d x \geq \\
& C_{1} \int_{\Omega_{+}}\left|\nabla u_{n}-\nabla u\right|^{p(x)} d x
\end{aligned}
$$

and

$$
\begin{aligned}
& \int_{\Omega_{-}}\left(A\left(x,\left|\nabla u_{n}\right|\right) \nabla u_{n}-A(x,|\nabla u|) \nabla u\right) \cdot\left(\nabla u_{n}-\nabla u\right) d x \geq \\
& C_{2} \int_{\Omega_{-}} \min \left\{1,\left(\left|\nabla u_{n}\right|+|\nabla u|\right)^{p(x)-2}\right\}\left|\nabla u_{n}-\nabla u\right|^{2} d x .
\end{aligned}
$$

Applying Hölder's inequality we obtain

$$
\int_{\Omega_{-}}\left|\nabla u_{n}-\nabla u\right|^{p(x)} d x \leq C\left(\int_{\Omega_{-}} \min \left\{1,\left(\left|\nabla u_{n}\right|+|\nabla u|\right)^{p(x)-2}\right\}\left|\nabla u_{n}-\nabla u\right|^{2} d x\right)^{\gamma},
$$

where $\gamma$ is a positive constant.

Relations (17), (18) and (19) imply our claim (16). This concludes the proof of Lemma.

Returning to the proof of Theorem 3.1, it remains to show that $\mathcal{E}$ satisfies the PalaisSmale condition. For this purpose, let $\left(u_{n}\right)$ be a sequence in $W_{0}^{1, p(x)}(\Omega)$ such that $\mathcal{E}\left(u_{n}\right) \rightarrow$ $c \in \mathbb{R}$ and $\mathcal{E}^{\prime}\left(u_{n}\right) \rightarrow 0$ in $W^{-1, p^{\prime}(x)}(\Omega)$. We first prove that

$$
\left(u_{n}\right) \text { is bounded in } W_{0}^{1, p(x)}(\Omega) \text {. }
$$


Using hypotheses (A4) and (f2) we have as $n \rightarrow \infty$

$$
\begin{aligned}
O(1)+o\left(\left\|u_{n}\right\|\right) & =\mathcal{E}\left(u_{n}\right)-\frac{1}{\mu} \mathcal{E}^{\prime}\left(u_{n}\right)\left(u_{n}\right) \\
& =\int_{\Omega}\left(\int_{0}^{\left|\nabla u_{n}\right|} s A(x, s) d s-\frac{1}{\mu} A\left(x,\left|\nabla u_{n}\right|\right)\left|\nabla u_{n}\right|^{2}\right) d x \\
& +\int_{\Omega}\left(f\left(x, u_{n}\right) u_{n}-F\left(x, u_{n}\right)\right) d x \\
& \geq\left(1-\frac{p_{+}}{\mu}\right) \int_{\Omega}\left(\int_{0}^{\left|\nabla u_{n}\right|} s A(x, s) d s\right) d x \\
& +\int_{\Omega}\left(f\left(x, u_{n}\right) u_{n}-F\left(x, u_{n}\right)\right) d x \\
& \geq\left(1-\frac{p_{+}}{\mu}\right) \int_{\Omega}\left(\int_{0}^{\left|\nabla u_{n}\right|} s A(x, s) d s\right) d x+O(1)
\end{aligned}
$$

Since $\mu>p_{+}$, we obtain

$$
\int_{\Omega}\left(\int_{0}^{\left|\nabla u_{n}\right|} s A(x, s) d s\right) d x=O(1) \quad \text { as } n \rightarrow \infty .
$$

Using now (A2) we deduce that

$$
\int_{\Omega}\left|\nabla u_{n}\right|^{p(x)}=O(1) \quad \text { as } n \rightarrow \infty
$$

which proves our claim (20).

Next, we show that if $\left(u_{n}\right)$ is bounded in $W_{0}^{1, p(x)}(\Omega)$ and satisfies for all $v \in W_{0}^{1, p(x)}(\Omega)$

$$
\mathcal{E}^{\prime}\left(u_{n}\right)(v)=\int_{\Omega} A\left(x,\left|\nabla u_{n}\right|\right) \nabla u_{n} \cdot \nabla v d x-\int_{\Omega} f\left(u_{n}\right) v d x=o(1) \quad \text { as } n \rightarrow \infty
$$

then $\left(u_{n}\right)$ is relatively compact. Using (f1), it is enough to show that a subsequence of $\left(\left|u_{n}\right|^{q(x)-1}\right)$ is convergent in $W^{-1, p^{\prime}(x)}(\Omega)$. By Sobolev embeddings for variable exponents, this property follows if we show that $\left(\left|u_{n}\right|^{q(x)-1}\right)$ is relatively compact in the variable exponent Lebesgue space $L^{N p(x) /[(N+1) p(x)-N]}(\Omega)$, which is the dual space of $L^{p^{*}(x)}(\Omega)$.

We first observe that, up to a subsequence,

$$
u_{n} \rightarrow u \in L^{p^{*}(x)}(\Omega) \quad \text { a.e. as } n \rightarrow \infty .
$$

Fix $\delta>0$. Applying the Egorov theorem, there exists an open set $\omega \subset \Omega$ with $|\omega|<\delta$ such that

$$
u_{n} \rightarrow u \quad \text { uniformly in } \Omega \backslash \omega .
$$

This shows that it is enough to prove that

$$
\left.\int_{\omega}|| u_{n}\right|^{q(x)-1}-\left.|u|^{q(x)-1}\right|^{N p(x) /[(N+1) p(x)-N]} d x
$$

can be made arbitrarily small.

Applying the Young inequality we find that there exists $C>0$ such that

$$
\int_{\omega}\left(|u|^{q(x)-1}\right)^{N p(x) /[(N+1) p(x)-N]} d x \leq C \int_{\omega}\left(|u|^{p^{*}(x)}+1\right) d x,
$$

which can be made as small as we wish, by choosing $\delta>0$ small enough. 
Fix $\varepsilon>0$. Since $q(x)<p^{*}(x)$, there exists $C_{e} p>0$ such that for all $n$

$$
\int_{\omega}\left(\left|u_{n}\right|^{q(x)-1}\right)^{N p(x) /[(N+1) p(x)-N]} d x \leq \epsilon \int_{\omega}\left|u_{n}\right|^{p^{*}(x)} d x+C_{\varepsilon}|\omega| .
$$

Using now (20) in combination with Sobolev embeddings for variable exponents we obtain

$$
\int_{\omega}\left(\left|u_{n}\right|^{q(x)-1}\right)^{N p(x) /[(N+1) p(x)-N]} d x \leq C \epsilon+C_{\varepsilon}|\omega|,
$$

which can be made small enough by choosing $\delta>0$ sufficiently small.

This concludes the proof of the Palais-Smale property and of Theorem 3.1.

\section{Proof of Theorem 3.2}

The spaces $W_{0}^{1, p(x)}(\Omega)$ and $W^{-1, p^{\prime}(x)}(\Omega)$ are reflexive and separable Banach spaces. Thus, by [12], there exist $\left\{e_{j}\right\} \subset X$ and $\left\{e_{j}^{*}\right\} \subset X^{*}$ such that

$$
W_{0}^{1, p(x)}(\Omega)=\overline{\operatorname{span}\left\{e_{j}: j=1,2, \ldots\right\}}, \quad W^{-1, p^{\prime}(x)}(\Omega)=\overline{\operatorname{span}\left\{e_{j}^{*}: j=1,2, \ldots\right\}},
$$

and

$$
\left\langle e_{i}, e_{j}^{*}\right\rangle= \begin{cases}1 & \text { if } \quad i=j \\ 0 & \text { if } \quad i \neq j\end{cases}
$$

where $\langle\cdot, \cdot\rangle$ denotes the duality product between $X$ and $X^{*}$. We define

$$
X_{j}=\operatorname{span}\left\{e_{j}\right\}, \quad Y_{k}=\bigoplus_{j=1}^{k} X_{j}, \quad Z_{k}=\overline{\bigoplus_{j=k}^{\infty} X_{j}} .
$$

The proof of Theorem 3.2 is based on the following basic critical point theorem.

Theorem 6.1. (Fountain Theorem, see [26]). Let $\mathcal{E} \in C^{1}(X)$ be an even functional, where $(X,\|\|$.$) is a separable and reflexive Banach space. Suppose that for every k \in \mathbb{N}$ large enogh, there exist $\rho_{k}>r_{k}>0$ such that

(i) $\inf \left\{\mathcal{E}(u): u \in Z_{k},\|u\|=r_{k}\right\} \rightarrow+\infty$ as $k \rightarrow+\infty$.

(ii) $\max \left\{\mathcal{E}(u): u \in Y_{k},\|u\|=\rho_{k}\right\} \leq 0$.

(iii) $\mathcal{E}$ satisfies the Palais-Smale condition for every $c>0$.

Then $\mathcal{E}$ has a sequence of critical values tending to $+\infty$.

The energy functional $\mathcal{E}$ is even and satisfies the Palais-Smale condition. We show that hypotheses (i) and (ii) in the statement of Theorem 6.1 are fulfilled.

Verification of (i). Fix $u \in Z_{k}$ with $\|u\|=r_{k}$, where $r_{k}>0$ will be specified later. Using hypotheses (A3), (A4) and (f1) we obtain

$$
\begin{aligned}
\mathcal{E}(u) & =\int_{\Omega}\left(\int_{0}^{|\nabla u(x)|} s A(x, s) d s-F(x, u)\right) d x \\
& \geq c_{1}|\nabla u|_{p(\cdot)}^{p_{-}}-c_{2} \int_{\Omega}\left(1+|u|^{q(x)}\right) d x \\
& \geq C_{1}\|u\|^{p_{-}}-C_{2} \max \left\{|u|_{q(\cdot)}^{q_{+}},|u|_{q(\cdot)}^{q_{-}}\right\}-C_{3} .
\end{aligned}
$$

Assuming that $\max \left\{|u|_{q(\cdot)}^{q_{+}},|u|_{q(\cdot)}^{q_{-}}\right\}=|u|_{q(\cdot)}^{q_{+}}$, we obtain the estimate

$$
\mathcal{E}(u) \geq C_{1}\|u\|^{p_{-}}-C_{2}|u|_{q(\cdot)}^{q_{+}}-C_{3} .
$$


Denote

$$
\alpha_{k}=\sup \left\{|u|_{L^{q(x)}(\Omega)} ;\|u\|=1, u \in Z_{k}\right\} .
$$

Then $\lim _{k \rightarrow \infty} \alpha_{k}=0$, see [10].

Since $u \in Z_{k}$, we deduce that

$$
\mathcal{E}(u) \geq C_{1}\|u\|^{p_{-}}-C_{2} \alpha_{k}^{q_{+}}\|u\|^{q_{+}}-C_{3} .
$$

Since $p_{-}<q_{+}$and $\alpha_{k} \rightarrow 0$, it follows that

$$
r_{k}:=\left(\frac{C_{2}}{C_{1}} \alpha_{k}^{p_{+}}\right)^{1 /\left(p_{-}-q_{+}\right)} \rightarrow+\infty \quad \text { as } k \rightarrow \infty .
$$

Taking $\|u\|=r_{k}$ we conclude that $\mathcal{E}(u) \rightarrow+\infty$, hence (i) is fulfilled.

Verification of (ii). Fix $u \in Y_{k}$ with $\|u\|=1$ and let $\rho_{k}$ be a positive number, which will be defined later. We have seen in relation (14) that there exists $\alpha>p_{+}$such that

$$
\mathcal{E}\left(\rho_{k} u\right) \leq C_{1}(u) \rho_{k}^{p_{+}}-C_{2} \rho_{k}^{\alpha}+C_{3},
$$

where $C_{1}, C_{2}, C_{3}$ are positive constants. Taking $\rho_{k}>r_{k}$ and using the fact that $\alpha>p_{+}$, we deduce that $\mathcal{E}\left(\rho_{k} u\right) \rightarrow-\infty$ as $k \rightarrow \infty$. This implies that

$$
\max \left\{\mathcal{E}(u) ; u \in Y_{k},\|u\|=\rho_{k}\right\} \leq 0,
$$

for every $\rho_{k}$ large enough. Applying the Fountain Theorem, we complete the proof of Theorem 3.2.

\section{Proof of Theorem 3.3}

For all $u \in W_{0}^{1, p(x)}(\Omega)$ we define

$$
I(u):=\int_{\Omega}\left(\int_{0}^{\mid \nabla u(x)} s A(x, s) d s\right) d x \text { and } J(u):=\int_{\Omega} F(x, u) d x .
$$

It follows that

$$
\lambda_{1}=\inf _{u \in W_{0}^{1, p(x)}(\Omega) \backslash\{0\}} \frac{I(u)}{J(u)} .
$$

Let $\left(u_{n}\right) \subset W_{0}^{1, p(x)}(\Omega)$ such that $u_{n} \rightarrow u$. We prove that

$$
I(u) \leq \liminf _{n \rightarrow \infty} I\left(u_{n}\right) \quad \text { and } \quad J(u)=\lim _{n \rightarrow \infty} J\left(u_{n}\right) .
$$

Since $\mathcal{E}_{0}^{\prime}$ is monotone, it follows that $I$ is convex. Therefore

$$
I\left(u_{n}\right) \geq I(u)+I^{\prime}(u)\left(u_{n}-u\right),
$$

hence $\liminf _{n \rightarrow \infty} I\left(u_{n}\right) \geq I(u)$.

Next, using assumptions (f1) and (f3) we deduce that there is a positive constant $C$ such that for all $(x, u) \in \Omega \times \mathbb{R}$

$$
|F(x, u)| \leq|u|^{p_{+}}+C|u|^{q(x)} .
$$

Since $p(x), q(x)<p^{*}(x)$ and $\left(u_{n}\right)$ is bounded in $W_{0}^{1, p(x)}(\Omega)$ we can assume that, up to a subsequence, $u_{n} \rightarrow u$ both in $L^{p_{+}}(\Omega)$ and in $L^{q(x)}(\Omega)$. Using relation $(22)$ we deduce that $J\left(u_{n}\right) \rightarrow J(u)$ as $n \rightarrow \infty$. 
Our hypotheses imply that for all $u \in W_{0}^{1, p(x)}(\Omega)$ with $\|u\|$ small enough we have

$$
I(u) \geq C_{1}\|u\|^{p_{+}}
$$

and

$$
\begin{aligned}
0 & \leq J(u)=\int_{\Omega} F(x, u) d x \leq C \int_{\Omega} \frac{|u|^{q(x)}}{q(x)} d x \\
& \leq C_{2}|u|_{q(\cdot)}^{q_{+}}+C_{3}|u|_{q(\cdot)}^{q_{-}} \leq C_{2}\|u\|^{q_{+}}+C_{3}\|u\|^{q_{-}} .
\end{aligned}
$$

Since $p_{+}<q_{-}$we deduce that

$$
\lim _{\|u\| \rightarrow 0} \frac{I(u)}{J(u)}=+\infty
$$

Using hypothesis (A5) and similar energy estimates as above we deduce that

$$
\lim _{\|u\| \rightarrow \infty} \frac{I(u)}{J(u)}=+\infty .
$$

Step 1. We prove that $\lambda_{1}>0$.

Our assumptions imply that $\lambda_{1} \geq 0$. Arguing by contradiction and supposing that $\lambda_{1}=0$, we find a sequence $\left(u_{n}\right) \subset W_{0}^{1, p(x)}(\Omega) \backslash\{0\}$ such that

$$
\lim _{n \rightarrow \infty} \frac{I\left(u_{n}\right)}{J\left(u_{n}\right)}=0 \text {. }
$$

We have already remarked that

$$
\frac{I(u)}{J(u)} \geq \frac{C_{1} \min \left\{\|u\|^{p_{+}},\|u\|^{p_{-}}\right\}}{C_{2}\|u\|^{q_{+}}+C_{3}\|u\|^{q_{-}}} .
$$

Since $p_{+}<q_{-}$, relations (25) and (26) imply that $\left(u_{n}\right)$ is unbounded. Using now (24) we contradict our assumption (25).

Step 2. We show that problem (4) has a solution for $\lambda=\lambda_{1}$.

Let $\left(u_{n}\right) \subset W_{0}^{1, p(x)}(\Omega) \backslash\{0\}$ be such that

$$
\left.\lim _{n \rightarrow \infty} \frac{I\left(u_{n}\right)}{J\left(u_{n}\right)}=\right] \lambda_{1} .
$$

Using $(21)$ we deduce that $\left(u_{n}\right)$ is bounded. Thus, up to a subsequence,

$$
u_{n} \rightarrow u \quad \text { in } W_{0}^{1, p(x)}(\Omega) .
$$

Assuming now that $u=0$, relation (21) shows that $J\left(u_{n}\right) \rightarrow 0$. Thus, by (27), we also have $I\left(u_{n}\right) \rightarrow 0$. But for all $u \in W_{0}^{1, p(x)}(\Omega)$

$$
I(u) \geq C_{1} \min \left\{\|u\|^{p_{+}},\|u\|^{p_{-}}\right\} .
$$

We deduce that $\left\|u_{n}\right\| \rightarrow 0$. Using now relation (24) we obtain a contradiction. This proves that $u \neq 0$. Using now (21) we conclude that $I(u)=\lambda_{1} J(u)$, hence $\lambda_{1}$ is an eigenvalue of problem (4).

Step 3. Every $\lambda>\lambda_{1}$ is an eigenvalue of problem (4).

Fix $\lambda>\lambda_{1}$. The energy functional associated to problem (4) is

$$
\mathcal{E}_{\lambda}(u):=I(u)-\lambda J(u) .
$$


Relation (24) shows that $\mathcal{E}_{\lambda}$ is coercive, that is, $\mathcal{E}_{\lambda}(u) \rightarrow+\infty$ as $\|u\| \rightarrow \infty$. But, by (21), $\mathcal{E}_{\lambda}$ is lower semi-continuous, hence it has a global minimizer $w \in W_{0}^{1, p(x)}(\Omega)$. On the other hand, since $\lambda>\lambda_{1}$, there exists $v \in W_{0}^{1, p(x)}(\Omega)$ such that

$$
\lambda_{1}<\frac{I(v)}{J(v)}<\lambda .
$$

This shows that $\mathcal{E}_{\lambda}(v)<0$, so $\mathcal{E}_{\lambda}(w)<0$. We conclude that $w \neq 0$ and $w$ is a critical point of $\mathcal{E}_{\lambda}$, hence a nontrivial solution of problem (4).

Acknowledgements. The author acknowledges the support by the Slovenian Research Agency grants P1-0292, J1-4144, and J1-5435.

\section{REFERENCES}

[1] M.J. Ablowitz, B. Prinari, and A.D. Trubatch, Discrete and Continuous Nonlinear Schrödinger Systems, Cambridge Univ. Press, Cambridge, 2004.

[2] A. Ambrosetti, P. Rabinowitz, Dual variational methods in critical point theory and applications, $J$. Functional Analysis 14 (1973), 349-381.

[3] G. Afrouzi, M. Mirzapour, V. Rădulescu, Existence and multiplicity results for anisotropic stationary Schrödinger equations, Atti Accad. Naz. Lincei Cl. Sci. Fis. Mat. Natur. Rend. Lincei Mat. Appl. 25 (2014), 91-108.

[4] T. Bartsch, Infinitely many solutions of a symmetric Dirichlet problem, Nonlinear Anal. 20 (1993), $1205-1216$

[5] Y. Chen, S. Levine, M. Rao, Variable exponent, linear growth functionals in image restoration, SIAM J. Appl. Math. 66 (2006), 1383-1406.

[6] P.G. Ciarlet, Linear and Nonlinear Functional Analysis with Applications, Society for Industrial and Applied Mathematics (SIAM), Philadelphia, 2013.

[7] L. Diening, P. Harjulehto, P. Hästö, M. Ruzicka, Lebesgue and Sobolev Spaces with Variable Exponents, Springer, Heidelberg, 2011.

[8] D.E. Edmunds, J. Rákosník, Density of smooth functions in $W^{k, p(x)}(\Omega)$, Proc. Roy. Soc. London Ser. A 437 (1992), 229-236.

[9] D.E. Edmunds, J. Rákosník, Sobolev embedding with variable exponent, Studia Math. 143 (2000), 267-293.

[10] X.L. Fan, Solutions for $p(x)$-Laplacian Dirichlet problems with singular coefficients, J. Math. Anal. Appl. 312 (2005), 464-477.

[11] X.L. Fan, X.Y. Han, Existence and multiplicity of solutions for $p(x)$-Laplacian equations in $\mathbb{R}^{\mathbb{N}}$, Nonlinear Anal. 59 (2004), 173-188.

[12] X.L. Fan, Q.H. Zhang, Existence of solutions for $p(x)$-Laplacian Dirichlet problems, Nonlinear Anal. 52 (2003), 1843-1852.

[13] T.C. Halsey, Electrorheological fluids, Science 258 (1992), 761-766.

[14] O. Kováčik, J. Rákosník, On the spaces $L^{p(x)}(\Omega)$ and $W^{1, p(x)}$, Czechoslovak Math. J. 41 (1991), 592618.

[15] N. Laskin, Fractional quantum mechanics and Lévy path integrals, Phys. Lett. A268 (2000), 298-305.

[16] M. Mihăilescu, P. Pucci, V. Rădulescu, Eigenvalue problems for anisotropic quasilinear elliptic equations with variable exponent, J. Math. Anal. Appl. 340 (2008), 687-698.

[17] M. Mihăilescu, V. Rădulescu, On a nonhomogeneous quasilinear eigenvalue problem in Sobolev spaces with variable exponent, Proceedings Amer. Math. Soc. 135 (2007), 2929-2937.

[18] J. Musielak, Orlicz Spaces and Modular Spaces, Springer-Verlag, Berlin, 1983.

[19] H. Nakano, Modulared Semi-ordered Linear Spaces, Maruzen, Tokyo, 1950.

[20] W. Orlicz, Über konjugierte Exponentenfolgen, Studia Math. 3 (1931), 200-211.

[21] M. Reed, B. Simon, Methods of Modern Mathematical Physics IV. Analysis of Operators, Academic Press, New York, 1978. 
[22] M. Ruzicka, Electrorheological Fluids. Modeling and Mathematical Theory, Lecture Notes in Mathematics 1748, Springer-Verlag, Berlin, 2000.

[23] J. Simon, Régularité de la solution d'une équation non linéaire dans $\mathbb{R}^{n}$, in Journées d'Analyse Non Linéaire, P. Benilan and J. Robert eds., Lecture Notes in Math., 665, Springer, Berlin, 1978, 205-227.

[24] W.A. Strauss, Existence of solitary waves in higher dimensions, Comm. Math. Phys. 55 (1977), 149-162.

[25] C. Sulem, P.L. Sulem, The Nonlinear Schrödinger Equation. Self-focusing and Wave Collapse, Applied Mathematical Sciences, Vol. 139, Springer-Verlag, New York, 1999.

[26] M. Willem, Minimax Theorems, Birkhäuser, Boston, 1996.

[27] V. Zhikov, Averaging of functionals of the calculus of variations and elasticity theory, Math. USSR-Izv. 29 (1987), 675-710.

[28] V. Zhikov, On Lavrentiev's phenomen, Rus. J. Math. Phys. 3 (1995), 249-269.

Faculty of Education, and Faculty of Mathematics and Physics, University of Ljubluana \& Institute of Mathematics, Physics and Mechanics, 1000 Luubluana, Slovenia

E-mail address: dusan.repovs@guest.arnes.si 